\title{
Upper Gastrointestinal Hemorrhage from Hepatic Artery Pseudoaneurysm Secondary to Trauma: A Case Report
}

\author{
Xiaofang Luo Shiyun Tan Junyu Wang Cuijuan Qian \\ Department of Digestive System, Renmin Hospital of Wuhan University, Wuhan, PR China
}

\section{Key Words}

Pseudoaneurysm • Upper gastrointestinal hemorrhage •

Angiography $\cdot$ Embolization

\begin{abstract}
Objective: We report a rare but serious case of upper gastrointestinal hemorrhage from hepatic artery pseudoaneurysm. Clinical Presentation and Intervention: An 11-yearold child with a history of hepatic trauma 6 months before was admitted for hematemesis and melena. Repeat ultrasound examination showed a $3.6 \times 2.8 \mathrm{~cm}$ anechoic area with clear border in the right hepatic lobe. Selective angiography confirmed the presence of a pseudoaneurysm of the right hepatic artery. Transcatheter arterial embolization was used to successfully treat the pseudoaneurysm. Conclusion: This report shows that hepatic artery pseudoaneurysm should be considered as a possible cause of upper gastrointestinal hemorrhage following known hepatic injury. A high index of suspicion, repeated Doppler ultrasound and timely selective angiography are required for diagnosis. Embolotherapy is the treatment of choice.
\end{abstract}

Copyright $\odot 2010$ S. Karger AG, Base

\section{Introduction}

Hepatic artery pseudoaneurysm (HAP), which was first described as an uncommon complication of trauma in 1984 by Sandblom [1], is a rare and serious cause of up- per gastrointestinal (UGI) hemorrhage [2]. It has been reported in relation to iatrogenic and traumatic cases, arteriosclerotic disease, vasculitis, pancreatitis and cholecystitis [3-6]. We herein present a case of posttraumatic HAP associated with UGI hemorrhage in an 11-year-old child.

\section{Clinical Presentation and Intervention}

An 11-year-old boy was admitted for hematemesis and melena for 1 week on March 30, 2006. One week before, he had been treated for hematemesis at a general hospital with blood transfusions, intravenous omeprazole and antibiotic but developed recurrent hematemesis and melena and was transferred to our hospital. He had a history of accidental hepatic trauma named hepatorrhexis which was caused by a hit of a handcart's bar and had undergone surgery for hepatic repair on October 20,2005. No further details about the surgical procedure were available.

On admission, he was hemodynamically stable, and his blood pressure was $110 / 60 \mathrm{~mm} \mathrm{Hg}$ with a heart rate of $100 \mathrm{bpm}$. Abdominal examination revealed no unusual findings except for an epigastric scar. The child had no fever or jaundice. Laboratory tests revealed moderate anemia (red blood cells $3.1 \times 10^{12} / 1$, hemoglobin $81 \mathrm{~g} / \mathrm{l}$, mean corpuscular hemoglobin concentration $303 \mathrm{~g} / \mathrm{l}$ ). Abdominal ultrasound (US) identified no abnormality. Upper endoscopy only showed superficial gastritis. The admitting diagnosis was UGI hemorrhage and iron deficiency anemia.

After admission, the patient was given blood transfusions, hemostasis therapy, intravenous omeprazole and parenteral nutritional support. Despite these treatments, the child continued to have recurrent hematemesis and melena. Emission computed tomography (CT) showed suspicious bleeding lesions in the left upper quadrant of the abdomen (probably stomach), so a second UGI endoscopy was performed, which revealed 3 scattered fresh

\section{KARGER}

Fax +4161306 1234 E-Mail karger@karger.ch www.karger.com
(C) 2010 S. Karger AG, Basel

1011-7571/10/0196-0493\$26.00/0

Accessible online at:

www.karger.com/mpp
Shiyun Tan

Department of Digestive System

Renmin Hospital of Wuhan University

238 Republic Road, Wuhan 430060 (PR China)

Tel./Fax +86278804 1911, E-Mail tanshiyun@medmail.com.cn 
Fig. 1. a An 11-year-old child with traumatic pseudoaneurysm (white arrow). Selected image from US. b Selected image from transcatheter hepatic angiogram examination. c Selected image from transcatheter hepatic angiogram examination; transcatheter arterial embolization showed the absence of the pseudoaneurysm (white arrow). d US showed the absence of the anechoic area 9 days after transcatheter arterial embolization.
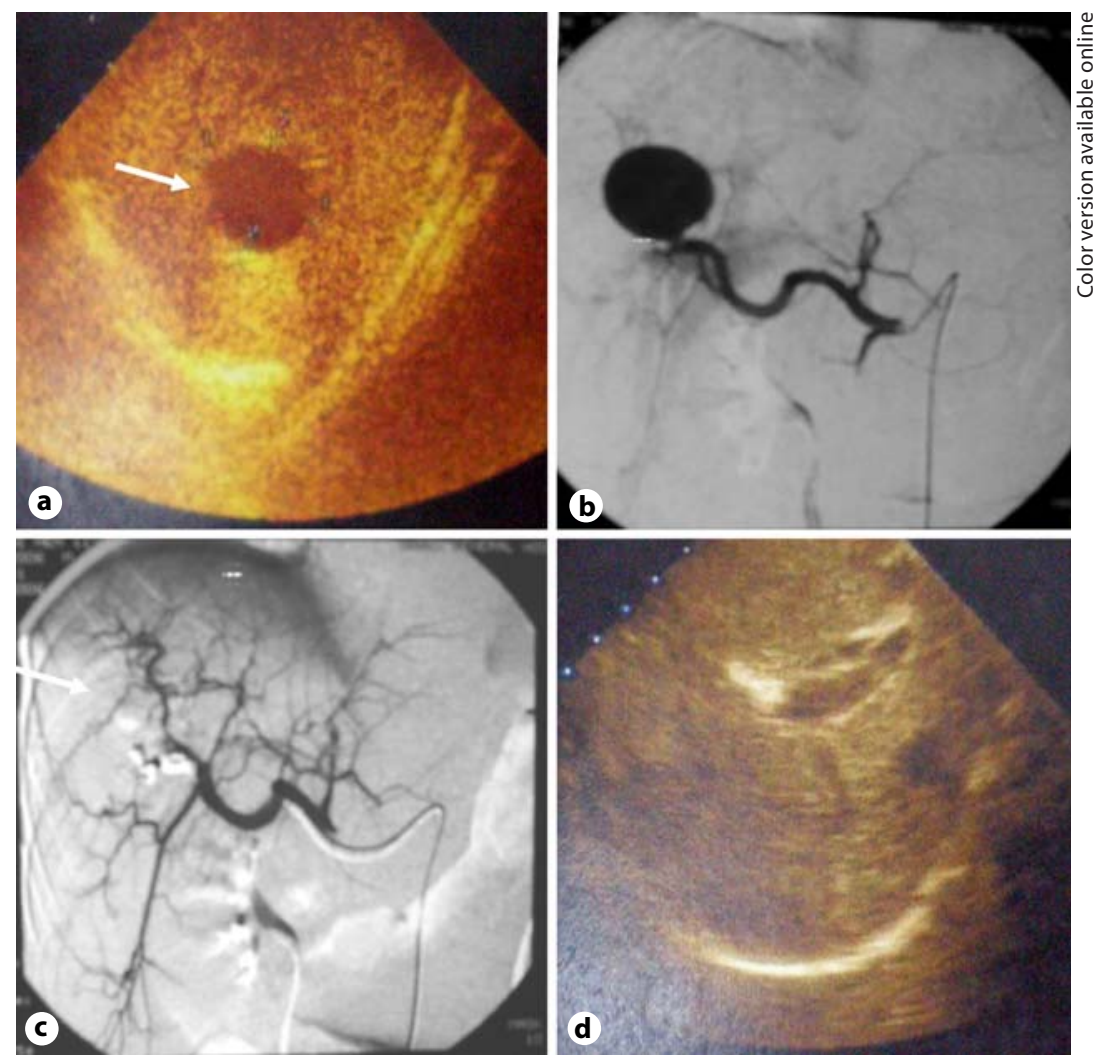

bleeding spots in the fundus, and the patient underwent titanium hemoclipping with a presumptive diagnosis of Dieulafoy disease.

Four days later, the child had a severe hemorrhage relapse, with approximately hematemesis of $300 \mathrm{ml}$ and melena of $200 \mathrm{ml}$, and his blood pressure quickly decreased to $90 / 60 \mathrm{~mm} \mathrm{Hg}$. Emergency blood transfusion and intravenous fluid infusion were used to keep him stable. A repeat US examination of the abdomen was performed and revealed a $3.6 \times 2.8 \mathrm{~cm}$ anechoic area with clear border in the right hepatic lobe which showed arterial flow by color Doppler flow imaging (fig. 1a). The portal vein diameter was normal. These findings suggested an aneurysm. Selective celiac arteriography confirmed the presence of a pseudoaneurysm of the right hepatic artery (fig. 1b) on April 13, 2006, and the next day, with use of coils, transcatheter arterial embolization was performed, with successful occlusion of the pseudoaneurysm (fig. 1c). The patient recovered uneventfully without further bleeding. Nine days later US showed the absence of the anechoic area (fig. 1d). The boy was discharged without any residual symptoms 11 days afterwards. At follow-up 2 years and 6 months later, the patient remained asymptomatic and was leading a normal life.

\section{Discussion}

UGI hemorrhage from a HAP is a rare but serious condition. In the majority of cases reported it was described as hemobilia $[1,4]$. A pseudoaneurysm is a pulsatile he- matoma due to a leakage of blood through a tear or disruption of the arterial wall and blood is contained only by hepatic parenchyma or surrounding hematoma. The HAP can rupture into the biliary system, causing hematobilia with hematemesis and/or melena. The causes of HAP include trauma, liver biopsy, infection or inflammation due to septic emboli or pancreatitis, radiofrequency ablation therapy, laparoscopic cholecystectomy and liver transplantation [2-4]. We believe the most likely sequence of events in our patient was that the pseudoaneurysm developed after the hepatic injury and then ruptured into the bile ducts, resulting in hemobilia.

Hemorrhage from a HAP is life-threatening, with a mortality rate of $9 \%$ in a recent series [5]. The high morbidity and mortality associated with pseudoaneurysms emphasize the importance of early diagnosis and treatment. The diagnosis is frequently delayed because it is unsuspicious presenting as fever, bile leak, hepatic dysfunction, abdominal pain, hematemesis, melena, anemia, hypovolemia or jaundice. The classic Quincke triad (UGI hemorrhage, upper abdominal pain and jaundice) occurred individually in 73, 52 and $30 \%$ of cases, with all 3 components occurring in only $22 \%$ of patients [6]. In our patient, the single mode of presentation was UGI hemor- 
rhage with no pain or jaundice. Nevertheless, the history of hepatic trauma and hematemesis with melena were highly suggestive of hemobilia from a HAP.

Selective arteriography is the standard and the most sensitive test for diagnosis. One previous report showed that $100 \%$ of HAPs were detected by selective arteriography in a series of 10 patients, compared with only $67 \%$ detected by CT and 33\% detected by Doppler US [7]. A pseudoaneurysm can sometimes be diagnosed with color Doppler US [3], which is also useful for follow-up assessment of an embolized aneurysm. In our patient, only the second US showed an anechoic area which was suggestive of an aneurysm. It was suspected that the first US was performed when the pseudoaneurysm was just evacuated due to bleeding. By contrast, the second US was obtained when the pseudoaneurysm had refilled after treatment of the patient with blood transfusion and intravenous fluids. Bleeding is often intermittent and it may be necessary to repeat the US. Occasionally a large aneurysm may be diagnosed by CT or magnetic resonance imaging [8]. Although HAP has been incidentally detected by endoscopic retrograde cholangiopancreatography, this procedure is not routinely recommended. Angiography is still the standard diagnostic choice that also provides accurate localization and an arterial road map essential for planning management.

Although HAP can resolve spontaneously through thrombosis [3], because there is a high risk of rupture, embolization or surgery are advocated as treatment choices when a HAP is detected. Angiographic emboliza- tion is an effective method of treatment, with a reported success rate of $80-100 \%[9,10]$. For high-risk patients, embolization is preferred and is well tolerated by the liver, provided the portal vein is patent $[9,10]$. A variety of embolic agents, such as gelatin sponge, polyvinyl alcohol, coils, N-butyl-cyanoacrylate and thrombin, have been employed for embolization $[9,10]$. Arterial stents and detachable silicone balloons have also been utilized. The choice of embolic material depends on the site and the size of the vessels to be occluded and on the characteristics of the lesion. In this case, we chose coils and treated the pseudoaneurysm successfully. Surgical intervention may be required when embolization fails, or if it follows orthotopic liver transplantation or an associated pseudocyst [11]. Hepatic lobectomy and debridement accompanied by ligation of the pseudoaneurysm are the two surgical approaches that have been successfully employed [4, $12]$.

\section{Conclusion}

This report shows that HAP should be considered as a possible cause of UGI hemorrhage following known hepatic injury. A high index of suspicion and repeated Doppler US when necessary with a timely selective arteriography are required for diagnosis. Embolization is an effective therapy, but surgical intervention may be needed if embolization fails.

\section{References}

1 Sandblom P: Hemorrhage into the biliary tract following trauma: 'traumatic hemobilia’. Surgery 1948;24:571-586.

$\checkmark 2$ Duce MN, Soyupak S, Inal M, Bijnokay F: Spontaneous thromsbosis of a post-traumatic hepatic artery pseudoaneurysm with arterio-biliary fistula in a child: helical CT diagnosis. Pediatr Radiol 2002;32:95.

$\checkmark 3$ Poon R, Tuen H, Yeung C, et al: GI haemorrhage from fistula between right hepatic artery pseudoaneurysm and the duodenum secondary to acute cholecystitis. Gastrointest Endosc 2000;51:491-493.

$\checkmark 4$ Croce MA, Fabian TC, Spiers JP, Kudsk KA: Traumatic hepatic artery pseudoaneurysm with hemobilia. Am J Surg 1994;168:235238 . $\checkmark 5$ Lumsden AB, Mattar SG, Allen RC, Bacha EA: Hepatic artery aneurysms: the management of 22 patients. J Surg Res 1996;60:345350.

6 Green MH, Duell RM, Johnson CD, Jamieson NV: Haemobilia. Br J Surg 2001;88:773786.

7 Tobben PJ, Zajko AB, Sumkin JH, Bowen A, Fuhrman CR, Skolnick ML: Pseudoaneurysms complicating organ transplantation: roles of CT, duplex sonography, and angiography. Radiology 1988;169:65-70.

$\checkmark 8$ Zalcman M, Matos C, Van-Gansbeke D, Engelholm L, de-Toeuf J, Struyven J: Hepatic artery aneurysm: CT and MR features. Gastrointest Radiol 1987;12:203-207.

$\checkmark 9$ Salam TA, Lumsden AB, Martin LG, Smith RB: Nonoperative management of visceral aneurysms and pseudoaneurysms. Am J Surg 1992;164:215-219.
0 Reber PU, Baer HU, Patel AG, Wildi S, Triller J, Buchler MW: Superselective microcoil embolization: treatment of choice in highrisk patients with extrahepatic pseudoaneurysms of the hepatic arteries. J Am Coll Surg 1998;186:325-330.

11 Fistouris J, Herlenius G, Bäckman L, Olausson M, Rizell M, Mjörnstedt L, Friman S: Pseudoaneurysm of the hepatic artery following liver transplantation. Transplant Proc 2006;38:2679-2682.

12 McGehee RN, Townsend CM, Thompson JC, et al: Traumatic hemobilia. Ann Surg 1974; 179:311-315. 